\title{
The IAU Strategic Plan for 2020-2030: OAO
}

\author{
Ewine F. van Dishoeck ${ }^{1}$ and Debra Meloy Elmegreen ${ }^{2}$ \\ ${ }^{1}$ Leiden Observatory, \\ P.O. Box 9513, NL-2300 RA, Leiden, the Netherlands \\ email: ewine@strw.leidenuniv.nl \\ ${ }^{2}$ Dept. of Physics \& Astronomy, Vassar College, \\ Poughkeepsie, NY 12604 USA \\ email: elmegreen@vassar.edu
}

\begin{abstract}
The IAU Strategic Plan for 2020-2030 presents an overview of all of the activities of the IAU along with priorities, key goals, mandates, and specific actions. Here future plans and goals are outlined for the Office of Astronomy for Development (OAO).
\end{abstract}

Keywords. editorials, notices

\section{Introduction}

The first formal Strategic Plan of the International Astronomical Union for the decade 2010-2020, was focused on the then-new Office of Astronomy for Development (OAD), which uses astronomy in developing countries to impact UN Sustainable Development Goals (SDGs). The IAU Executive Committee (EC) decided that the new Strategic Plan (SP) for 2020-2030 should encompass all activities of the IAU. The EC working group charged to write the new SP included Ewine van Dishoeck, Debra Elmegreen, Piero Benvenuti and Renée Kraan-Korteweg and received extensive input from its membership and the various Offices, in particular from Sze-leung Cheung and his team on the OAO part.

The final Strategic Plan 2020-2030 was presented for adoption as Resolution A1 at this XXXth GA, where it was approved at the second Business Meeting (Fig. 1). The revised mission of the IAU is to "promote and safeguard astronomy in all its aspects (including research, communication, education, and development) through international cooperation," where the additional parenthetical aspects make explicit that the IAU has branched out beyond its original purpose of fostering scientific communication and exchange of ideas among professional astronomers.

\section{OAO and other Offices of the IAU}

The 3 current offices of the IAU were formed within the last decade to carry out additional aspects of IAU activities beyond the advancement of astronomy. The Office for Astronomy Outreach (OAO) is a partnership with the National Astronomical Observatory of Japan, located at the Mitaka campus in Tokyo, and focuses on engaging with the public, providing access to astronomical information and astronomy communication. It maintains a network of National Outreach Contacts (NOCs) and amateur groups. The OAO interacts closely with the OAD, hosted in Cape Town, South Africa, which focuses on the use of astronomy for development by capitalizing on the field's scientific, technological and cultural links and its impacts on society. The Office of Young Astronomers, hosted by the Norwegian Academy of Science and Letters, focuses on the training of young astronomers at University level, and organizes the International School for Young Astronomers (ISYA). A new office is proposed: the Office of Astronomy for 


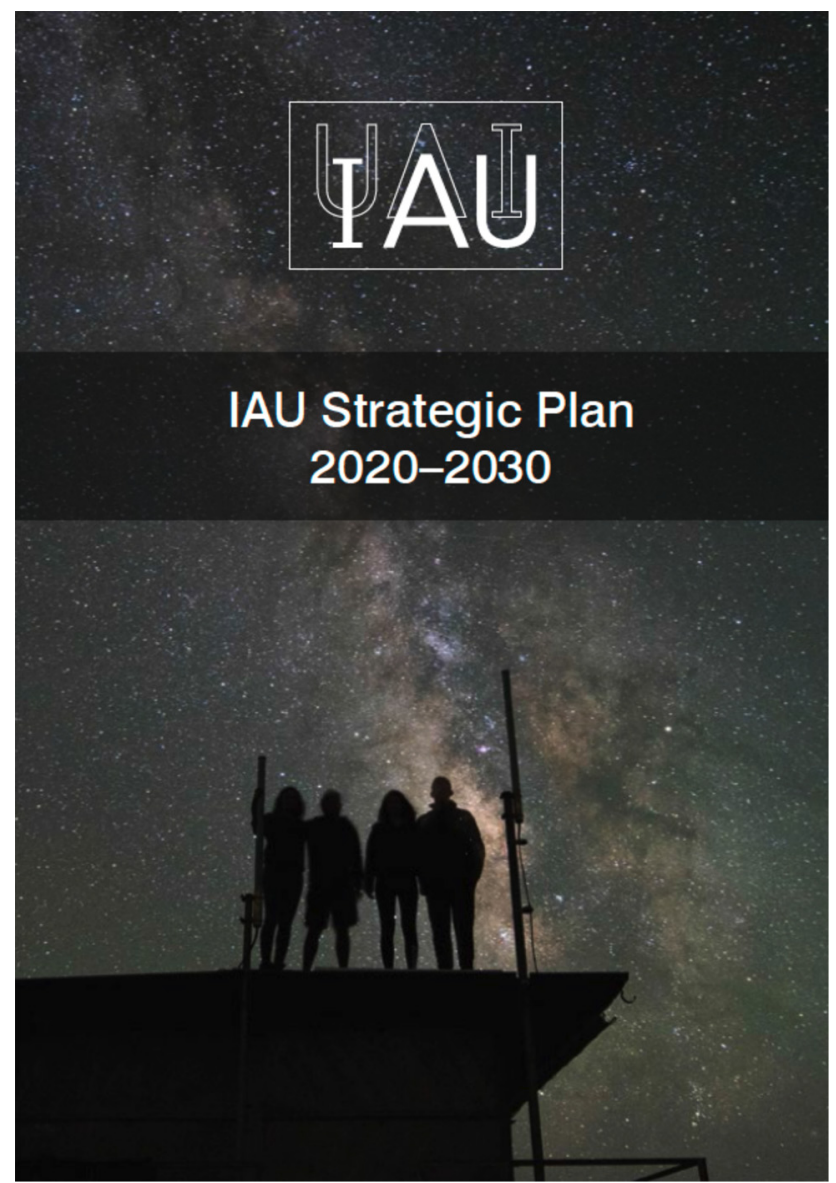

Figure 1. Cover of the new IAU strategic plan, illustrating the importance of outreach, engaging with the general public and dark skies.

Education (OAE) will focus on providing training and resources for using astronomy as a stimulus for teaching and education from elementary to high school level (astronomy and science education). Each of these offices has its own strategic plan, and each office has a liaison on the EC to ensure that the strategic plans align with the overall goals of the IAU. Sometimes activities span more than one office, so having connections with the EC helps coordinate overlapping activities.

\section{Office for Astronomy Outreach (OAO)}

The Office for Astronomy Outreach (OAO) coordinates public outreach activities and communication of science to the public. Its long-term vision is: that all people throughout the world will have access to knowledge of frontline astronomy; that all countries will have good access to astronomical research, culture and experiences to help build a literate society; and that astronomers are a strong part of the global citizenship.

The central OAO function is accessibility. The OAO generally does not create outreach material itself, but works with the IAU and other organisations to increase the impact of its activities. Outreach is a strong component of many different units within the IAU, and the OAO interfaces with all of them. For example, Commission C2, 'Communicating Astronomy with the Public' (CAP), addresses astronomy communication and outreach 
issues, with the OAO editing the CAP journal as well as an Astronomy Outreach Newsletter. Also, the OAO works with the OAD to conduct numerous actions related to public outreach in order to achieve SDGs. By working together, both offices can maximise their synergies in the same area but with different goals. The OAO also takes part in EPO-like (Education and Public Outreach) activities, thus providing an interface with the newly proposed Office of Astronomy for Education.

To reach its long-term goals, OAO activities will include the provision of easily accessible public-friendly information on astronomical terminologies and objects in the Universe. To help reach larger audiences, the OAO will expand its translation network in the coming decade to manage and distribute astronomical results in several different languages. Activities to connect professional and amateur astronomers will be strengthened in the coming decade. The OAO also coordinates some of the worldwide citizen-science projects and campaigns, such as the public exoplanet naming competition in 2015 2016. Taken together, the $\mathrm{OAO}$ activities contribute to providing inclusive resources globally and achieving a more diverse astronomical community.

The network of National Outreach Coordinators, maintained by the OAO, can coordinate and advance projects in their country, as was done with great success during the 2009 International Year of Astronomy, and is being done in 2019 for the IAU100 activities. The NOCs are also the formal point of contact for engagement with amateur astronomy groups within each country. Furthermore, NOCs can play a role in helping to spread the word about the dark and quiet sky initiatives and to promote citizen science projects. In the coming years, the NOC system will be redefined, restructured and enlarged to ensure its effectiveness across the world.

In summary, the goals of the OAO for the next decade are:

- Increase the network of NOCs; restructure and ensure their effectiveness.

- Facilitate international communication through exchanges and translations.

- Provide open databases and public-friendly access to astronomical information.

- Encourage communication of science and critical thinking through IAU member public engagement, professional-amateur, and citizen science activities.

- Promote dark skies and the pale blue dot message

The IAU is grateful to the OAO staff and the many people who have contributed to the achievements of the OAO so far, especially the National Outreach Coordinators. We encourage colleagues and early career astronomers to join the IAU and contribute to the OAO goals.

\section{References}

Astronomy for Development: Strategic Plan 2010-2020, https://www.iau.org/static/education/strategicplan_2010-2020.pdf IAU Strategic Plan 2020-2030, https://www.iau.org/static/education/strategicplan-2020-2030.pdf 